\title{
Kit Expression in Small Cell Carcinomas of the Lung: Effects of Chemotherapy
}

Giulio Rossi, M.D., Alberto Cavazza, M.D., Alessandro Marchioni, M.D., Mario Migaldi, M.D., Ph.D., Mario Bavieri, M.D., Nicola Facciolongo, M.D., Stefano Petruzzelli, M.D., Lucia Longo, M.D.,

Stefano Tamberi, M.D., Lucio Crinò, M.D.

Department of Pathologic Anatomy and Forensic Medicine, Section of Pathology (GR, MM), and Department of Respiratory Diseases (AM, MB, SP), University of Modena and Reggio Emilia; Operative Units of Pathology (AC) and Pneumology (NF), Ospedale S. Maria Nuova, AUSL Reggio Emilia; and Operative Unit of Oncology (LL, ST), Ospedale degli Infermi, Faenza, AUSL Ravenna; and Medical Oncology Division (LC), Ospedale Bellaria, Bologna, Italy

A significant number of small cell lung carcinomas shows overexpression of the proto-oncogene c-kit product, a tyrosine kinase known as Kit or CD117. This molecular pathway seems somewhat implicated in promoting the neoplastic growth of small cell lung carcinoma. The current pharmacological availability of its selective inhibitor, together with the promising clinical results in the management of CD117-positive neoplasms such as advanced gastrointestinal stromal tumors, aroused great interest among oncologists in also adopting this therapeutic strategy in other CD117-positive tumors. We evaluated a series of 27 small cell lung carcinomas, comparing the expression of $\mathrm{CD} 117$ of the primary naïve tumor (before first-line chemotherapy) with the expression of the same neoplasm after postchemotherapy relapse. All the patients underwent similar chemotherapeutic regimens (cisplatin/carboplatin plus etoposide). At diagnosis, 21 of 27 cases (78\%) showed strong immunoreactivity for CD117. Among these 21 originally positive tumors, CD117 remained overexpressed in 10 after relapse $(48 \%)$, whereas the other 11 cases became negative. No originally CD117-negative small cell carcinomas displayed immunoreactivity after chemotherapy. CD117 expression was not statistically correlated with overall survival, occurrence of chemoresistance, or clinical response to chemotherapy. We also evaluated CD117 expression in a series of 46

Copyright (C) 2003 by The United States and Canadian Academy of Pathology, Inc.

VOL. 16, NO. 10, P. 1041, 2003 Printed in the U.S.A

Date of acceptance: June 20, 2003.

Presented in part at the 39th Annual Meeting of the American Society of Clinical Oncology (ASCO), Chicago, Illinois, May 31-June 3, 2003.

Address reprint requests to: Giulio Rossi, M.D., Department of Pathologic Anatomy and Forensic Medicine, Section of Pathology, University of Modena and Reggio Emilia, via del Pozzo, 71-41100, Modena, Italy; fax: +39.059.4224820; e-mail: rossi.giulio@unimo.it.

DOI: $10.1097 / 01 . M P .0000089780 .30006 . D E$ surgically resected non-small cell lung carcinomas (8 squamous cell carcinomas, 10 adenocarcinomas, 5 pleomorphic carcinomas, 10 typical and 3 atypical carcinoids, and 10 large cell neuroendocrine carcinomas). Apart from small cell carcinomas, CD117 overexpression was observed in 6 of 10 large cell neuroendocrine carcinomas, whereas all the other histotypes resulted unstained. We speculate that loss of CD117 expression after chemotherapy in a high proportion of SCLC indicates that in this tumor, Kit unlikely represents the product of a constitutive mutation, as instead shown in gastrointestinal stromal tumors. Keeping this finding in mind, oncologists could re-test CD117 expression in relapsing small cell lung carcinomas in order to establish the best candidates for enrollment in ongoing clinical trials with Kit inhibitors. Practically speaking, CD117 may be helpful in discriminating between pulmonary high-grade neuroendocrine tumors and other histotypes, but pathologists should be aware that treated small cell lung carcinomas may remain unstained in a not insignificant number of cases.

KEY WORDS: CD117, c-kit, Immunohistochemistry, Lung, Small cell carcinoma, STI571.

Mod Pathol 2003;16(10):1041-1047

Small cell lung carcinoma accounts for 15 to $20 \%$ of all primary lung tumors (1). It is considered a systemic disease tending to disseminate early in the course of its natural history, and it is associated with the lowest 5 -year survival rate among all lung cancers (2-4). Despite modifications of drug compositions and doses in chemotherapeutic protocols and combinations of chemotherapy with chest and panencephalic preventive radiotherapy, little improvement has been achieved in patients' survival (2-4). Until now, combined chemotherapy has re- 
mained the mainstay in treating such tumors, but new approaches are clearly needed, especially in chemoresistant cases. In this regard, great expectations aroused by the observation that the autocrine/paracrine growth regulation pathway mediated by Kit/CD117, the proto-oncogene c-kitderived product, is strongly expressed in $\geq 70 \%$ of small cell carcinomas of the lung, both on cell lines and paraffin-embedded tissues (5-12). Kit is a Type III transmembrane tyrosine kinase receptor of 145 $\mathrm{kDa}$ that is structurally and functionally related to platelet-derived growth factor receptor (PDGFR) (5, $6,12,13)$. This membrane-bound protein is essential for the regulation of cell development and growth of some hematopoietic cell lines, mast cells, melanocytes, germ cells, and interstitial gastrointestinal cells of Cajal $(8,11-13)$. Its phosphorylation and dimerization, as a result of binding with steel factor or stem cell factor (SCF), provokes a cascade of intracytoplasmic signals that are deeply implicated in promoting and keeping neoplastic proliferation in malignancies of different histogenesis, especially in gastrointestinal stromal tumors, mast cell disorders, and acute myeloproliferative disorders and in a restricted subset of other solid malignancies, including seminomas and small cell lung carcinomas (11-20). The important therapeutic results using imatinib mesylate (formerly STI571, Gleevec; Novartis Pharmaceuticals Corp., East Hanover, NJ), a selective inhibitor of different tyrosine kinases that has been implicated in pathogenesis of chronic myeloid leukemia (Bcr-Abl) (21), gastrointestinal stromal tumors (Kit) (22-25), and dermatofibrosarcoma protuberans (PDGFR) (26), seem to provide an attractive base for the development of this biologic therapy in other Kit-positive neoplasms also. Although it is yet unanswered whether Kit expression in small cell lung carcinoma might be related to an activating (gain-of-function) mutational event, as instead demonstrated in the above pathologic conditions (22-26), preclinical in vitro studies reported that imatinib mesylate efficiently blocked neoplastic growth of small cell lung carcinoma cell lines (27-29). In light of these observations, we investigate by immunohistochemistry whether CD117 expression was modified by chemotherapy in a series of 27 patients with small cell carcinoma of the lung who underwent tumor biopsy before first-line chemotherapy and after relapse. Immunohistochemical results were then combined with clinical findings to analyze the value of CD117 immunostaining in predicting chemoresistance, clinical response, and overall survival in this specific setting. Moreover, a series of 46 surgically resected non-small cell lung tumors of various histotypes were tested for CD117 immunoreactivity to evaluate the possible diagnostic role of this marker in lung neoplasms.

\section{MATERIALS AND METHODS}

\section{Patient Selection and Immunohistochemistry}

From the files of the Section of Pathology of the University of Modena and Reggio Emilia and from the Operative Unit of Pathology of the Hospital S. Maria Nuova of Reggio Emilia, we collected 27 patients with small cell carcinoma of the lung who had biopsies obtained before and after postchemotherapy relapse between 1991 and 2001. At diagnosis, tissue specimens consisted of 20 bronchial biopsies, 2 transthoracic biopsies, and 5 pulmonary wedge resections, whereas relapsed tumors consisted of 20 bronchial biopsies, 1 transthoracic biopsy, and 6 lobectomies. All the patients underwent a similar first-line chemotherapeutic regimen (cisplatin/carboplatin plus etoposide). The second biopsy was obtained after at least three courses of chemotherapy. Extent of disease at diagnosis was assessed by fiberscopic bronchoscopy, chest radiograph, total-body CT scan, and bone scintigraphy. Tumor stage was assessed according to the twostage Veterans Administration Lung Study Group system (30). Response to treatment was assessed by total-body CT scan, and patients were stratified as nonresponders, partial responders, or complete responders, in agreement with the protocol of Therasse et al. (31).

The tumor samples were routinely fixed in $10 \%$ buffered formalin and then paraffin embedded. All the slides were reviewed at a multiheaded microscope by two pathologists (GR, AC). The cases were included in the study if they fulfilled the accepted histologic criteria for small cell lung carcinoma (32). For a better comprehension of the possible diagnostic role of CD117 expression in these tumors, 46 surgically resected non-small cell lung tumors (including 8 squamous cell carcinomas, 10 adenocarcinomas, 5 pleomorphic carcinomas, 10 typical and 3 atypical carcinoids, and 10 large cell neuroendocrine carcinomas) were randomly retrieved from the files of a single institution (the section of pathology of the University of Modena and Reggio Emilia) and subsequently tested for CD117 immunostaining.

In each case, $4-\mu \mathrm{m}$-thick sections were obtained from a representative block. Sections were air-dried overnight at $37^{\circ} \mathrm{C}$, then deparaffinized in xylene and rehydrated through a decreasing concentration of alcohol to water. Endogenous peroxidase activity was blocked by immersion for 10 minutes with $3 \%$ hydrogen peroxide $\left(\mathrm{H}_{2} \mathrm{O}_{2}\right)$ in methanol. Immunohistochemical staining was performed using a 1:200 dilution of the polyclonal antibody A4502 (DAKO, Glostrup, Denmark), without antigen retrieval. Incubation with the primary antibodies was accomplished with a modified avidin-biotin peroxidase technique using a commercial immunohistochem- 
ical autostainer (Ventana, Strasbourg, France); 3'3diaminobenzidine was used as the chromogen, and Harris hematoxylin, as the counterstain. Mast cells and fibroblasts served as positive internal controls for CD117, whereas appropriate sections of a gastrointestinal stromal tumor known to be strongly positive for CD117 were used as external positive control. Negative controls were included in each test by substituting the primary antibodies with nonimmune mouse IgG.

The percentage of positive cells (0: negative, $1+$ : 1 to $25 \%, 2+: 26$ to $50 \%$, and $3+:>50 \%$ ) and the intensity of staining (0: negative, 1 : weak, 2: moderate, 3: strong) were recorded in each case. A tumor was considered positive if $>50 \%$ of the neoplastic cells reacted with an intensity of $\geq 2+$ on the relevant subcellular localization (cytoplasmic and membranous distribution with or without dotlike accentuation). Clinical data were collected from pathological reports, clinical charts, and referring physicians or directly from the patient's families.

\section{Statistical Analysis}

The comparison between clinicopathologic parameters and immunohistochemical results was performed using contingency table methods. Results were tested for significance using the Pearson's $\chi^{2}$ test and Student's $t$ test and by means of one-way ANOVA analysis. Estimation of the cumulative distribution of the overall survival was calculated using the Kaplan-Meier method, and the logrank test was used for analysis. All calculations were performed with Statistical Package for Social Sciences (SPSS) release 8.00, and differences were considered significant at $P$ values of $<.05$.

\section{RESULTS}

The relevant clinical data and the immunohistochemical results are summarized in Table 1. Briefly, case series consisted of 27 patients ( 17 males and 10 females) with small cell carcinoma of the lung who underwent tumor biopsy both at diagnosis (on naive untreated cancer) and at the onset of chemoresistance after first-line chemotherapy (cisplatin plus etoposide in 18 cases and carboplatin plus etoposide in 9). All the patients but two were smokers. Age ranged from 29 to 82 years (mean, 66 y; median, 66 y). At diagnosis, 10 patients were clinically staged as having limited disease, whereas the other 17 had extensive disease. Follow-up was obtained in all the cases, ranging from 6 to 92 months (median, $22 \mathrm{mo}$ ). Twenty-four patients died of tumor progression, whereas three are alive with disease. Immunohistochemically, bronchial and bronchiolar epithelium and alveolar pneumocytes in

Table 1. Summary of the Clinical Data and Immunohistochemical Results for CD117 Expression in Small Cell Lung Carcinomas

\begin{tabular}{|c|c|c|c|c|c|c|c|c|}
\hline Case & Age (years) & Sex & Stage & $\begin{array}{l}\text { CD117-preCT } \\
\text { (intensity/\%) }\end{array}$ & $\begin{array}{c}\text { First Line } \\
\text { Chemotherapy }\end{array}$ & Clinical Response & $\begin{array}{c}\text { CD117-PostCT } \\
\text { (intensity/\%) }\end{array}$ & $\begin{array}{c}\text { Follow-Up } \\
\text { (months) }\end{array}$ \\
\hline 1 & 62 & M & $\mathrm{LD}$ & $3+/ 80 \%$ & Cis + Eto & PR & $-1-$ & 14, DOD \\
\hline 2 & 63 & $\mathrm{~F}$ & ED & $-1-$ & Carbo + Eto & NR & $-1-$ & 6, DOD \\
\hline 3 & 66 & $\mathrm{M}$ & $\mathrm{LD}$ & $3+/ 100 \%$ & Carbo + Eto & PR & $3+/ 80 \%$ & $11, \mathrm{DOD}$ \\
\hline 4 & 29 & $\mathrm{~F}$ & ED & $3+/ 100 \%$ & Cis + Eto & CR & $1+/ 10 \%$ & 45, DOD \\
\hline 5 & 75 & $\mathrm{~F}$ & $\mathrm{LD}$ & $3+/ 80 \%$ & Carbo + Eto & CR & $-1-$ & 42, DOD \\
\hline 6 & 56 & $\mathrm{~F}$ & $\mathrm{LD}$ & $-1-$ & Cis + Eto & PR & $-1-$ & 20, DOD \\
\hline 7 & 61 & $\mathrm{~F}$ & $\mathrm{LD}$ & $1+/ 10 \%$ & Carbo + Eto & CR & $-1-$ & $55, \mathrm{DOD}$ \\
\hline 8 & 59 & M & ED & $2+/ 60 \%$ & Cis + Eto & PR & $1+/ 3 \quad \%$ & $22, \mathrm{DOD}$ \\
\hline 9 & 65 & M & ED & $3+/ 70 \%$ & Cis + Eto & PR & $2+/ 80 \%$ & $30, \mathrm{DOD}$ \\
\hline 10 & 82 & M & ED & $3+/ 75 \%$ & Cis + Eto & CR & $1+/ 10 \%$ & $32, \mathrm{DOD}$ \\
\hline 11 & 73 & $\mathrm{~F}$ & ED & $3+/ 100 \%$ & Cis + Eto & NR & $3+/ 70 \%$ & $10, \mathrm{DOD}$ \\
\hline 12 & 59 & M & ED & $3+/ 100 \%$ & Carbo + Eto & NR & $1+/ 15 \%$ & 19, DOD \\
\hline 13 & 54 & $\mathrm{~F}$ & $\mathrm{LD}$ & $-1-$ & Cis + Eto & PR & $-1-$ & $11, \mathrm{DOD}$ \\
\hline 14 & 73 & M & ED & $3+/ 100 \%$ & Carbo + Eto & PR & $3+/ 100 \%$ & $34, \mathrm{DOD}$ \\
\hline 15 & 74 & M & ED & $2+/ 90 \%$ & Cis + Eto & PR & $-1-$ & $30, \mathrm{DOD}$ \\
\hline 16 & 71 & M & ED & $2+/ 80 \%$ & Carbo + Eto & NR & $1+/ 10 \%$ & $10, \mathrm{DOD}$ \\
\hline 17 & 71 & M & ED & $3+/ 100 \%$ & Cis + Eto & PR & $3+/ 90 \%$ & 20, DOD \\
\hline 18 & 75 & M & ED & $3+/ 100 \%$ & Cis + Eto & PR & $3+/ 100 \%$ & 22, DOD \\
\hline 19 & 63 & M & ED & $3+/ 100 \%$ & Cis + Eto & PR & $3+/ 80 \%$ & 19, DOD \\
\hline 20 & 57 & $\mathrm{M}$ & ED & $3+/ 100 \%$ & Cis + Eto & PR & $3+/ 100 \%$ & 11, DOD \\
\hline 21 & 65 & M & ED & $3+/ 100 \%$ & Cis + Eto & CR & $3+/ 100 \%$ & 33, DOD \\
\hline 22 & 73 & $\mathrm{M}$ & $\mathrm{LD}$ & $3+/ 100 \%$ & Cis + Eto & CR & $2+/ 80 \%$ & 92, AWD \\
\hline 23 & 73 & $\mathrm{~F}$ & $\mathrm{LD}$ & $3+/ 80 \%$ & Cis + Eto & NR & $1+/ 2 \quad \%$ & 8, DOD \\
\hline 24 & 60 & $\mathrm{~F}$ & $\mathrm{LD}$ & $3+/ 100 \%$ & Carbo + Eto & CR & $1+/ 3$ & 23, AWD \\
\hline 25 & 75 & $\mathrm{~F}$ & $\mathrm{LD}$ & $-1-$ & Carbo + Eto & $\mathrm{CR}$ & $-1-$ & 46, AWD \\
\hline 26 & 71 & M & ED & $3+/ 90 \%$ & Cis + Eto & PR & $-1-$ & $11, \mathrm{DOD}$ \\
\hline 27 & 72 & M & ED & $-1-$ & Cis + Eto & $\mathrm{RC}$ & $-1-$ & 51, DOD \\
\hline
\end{tabular}

F, female; M, Male; LD, limited disease; ED, extensive disease; Cis, cisplatin; Carbo, carboplatin; Eto, etoposid; CR, complete response; PR, partial response; NR, no response; DOD, dead of disease; AWD, alive with disease; CD117-preCT, CD117 expression before first line chemotherapy; CD117postCT, CD117 expression after first line chemotherapy. 
normal lung parenchyma adjacent to tumor remained completely unstained for CD117, whereas scattered positive mast cells and fibroblasts were noted in the interstitial stroma and in mesenchymal tissue around bronchi and bronchioles and served as positive internal controls. Among nonsmall cell lung neoplasms, including squamous cell carcinomas (8 cases), adenocarcinomas (10 cases), pleomorphic carcinomas (5 cases), and carcinoid tumors (10 typical and 3 atypical), no immunostaining was observed except in rare and scattered tumor cells in 2 of 10 adenocarcinomas. By contrast, strong and diffuse Kit overexpression was observed in 21 of 27 naive small cell lung carcinomas (78\%; Fig. 1) and in 6 of 10 large cell neuroendocrine carcinomas $(60 \%)$. In comparison with other non-small cell lung tumors, high-grade neuroendocrine carcinomas (small cell carcinomas and large cell neuroendocrine carcinomas) showed a statistically significant difference for Kit expression $(P<$ $.001 ; \chi^{2}$ test). Of the 21 positive small cell carcinomas, immunoreactivity was maintained after postchemotherapy relapse in only 10 (48\%), whereas no staining was observed in the remaining 11 (52\%; Fig. 2). The six patients who were negative at diagnosis remained negative also after postchemotherapy relapse. A statistically significant decrease of CD117 immunoreactivity after chemotherapy was found in regard to intensity staining $(P$ $=.006 ; \chi^{2}$ test) and percentage of positive tumor cells $(P=.003 ; t$ test $)$. Anecdotally, just one of the patients with large cell neuroendocrine carcinoma underwent surgical resection of the lung before and after chemotherapy, even if performing a different regimen (cisplatin plus gemcitabine), but both tumors strongly stained with CD117.

No significant difference in overall survival and response to chemotherapy was observed between CD117-positive and CD117-negative small cell lung

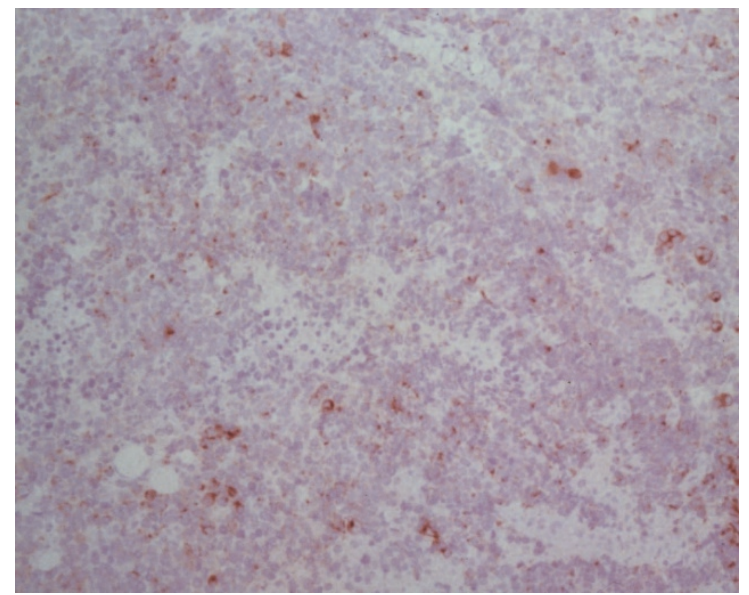

FIGURE 2. After first-line chemotherapy, the same patient underwent a transthoracic biopsy of the relapsed small cell lung carcinoma, but the tumor was essentially unstained for Kit protein (CD117, immunoperoxidase, $200 \times$ magnification)

carcinomas. As expected, stage (limited disease) and positive clinical response (partial or complete) correlated significantly with patient survival $(P=$ .045 and $P=.034$, respectively; log-rank test). Patients with CD117-positive tumors had more frequently extensive disease (15 of 17) in comparison to CD117-negative cases (6 of 10), although the difference did not reach statistical significance $(P=$ .153; $\chi^{2}$ test).

\section{DISCUSSION}

In a recent editorial, Carney (33) stated that "chemotherapy in advanced lung cancer has reached a plateau" and thus alternative therapeutic approaches are clearly required, especially in chemoresistant cases. In this view, understanding of lung cancer biology focused particular interest on several autocrine/paracrine growth loops that were
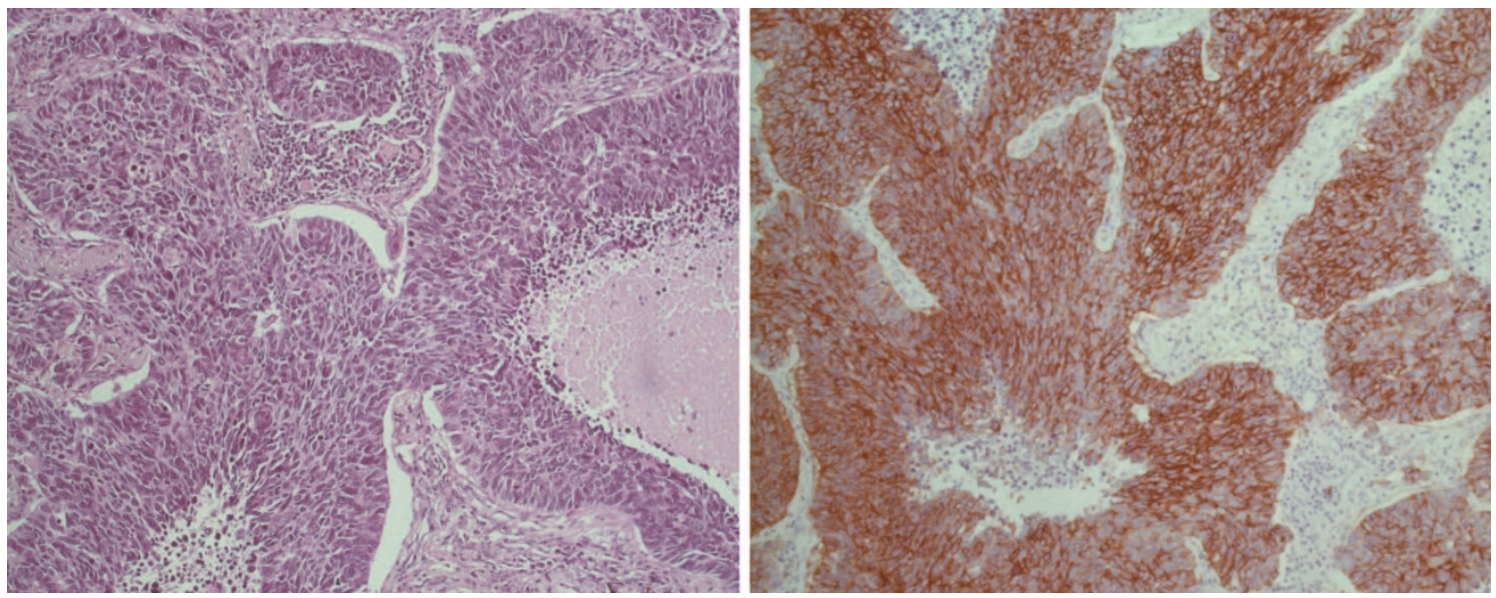

FIGURE 1. In this case (Case 24), a pulmonary wedge resection showed a small cell lung carcinoma (on the left; hematoxylin and eosin, 200× magnification). At diagnosis, the tumor overexpressed Kit protein (on the right; CD117, immunoperoxidase, 200 $\times$ magnification). 
thought to be somewhat implicated in their pathogenesis (34-38). Several works (6-17) demonstrated that $\geq 70 \%$ of small cell carcinomas of the lung coexpress Kit/CD117, the c-kit proto-oncogene-derived product, and its ligand stem cell factor (SCF). It is thus suggested that the Kit/SCF pathway promotes and keeps tumor cell proliferation in in vitro small cell lung carcinoma cell lines. Moreover, Krystal et al. (27), Wang et al. (28), and, more recently, Kijima et al. (29) elegantly demonstrated that small cell lung carcinoma cell line growth is significantly blocked by STI571/imatinib mesylate, a 2-phenylaminopyrimidine derivative inhibiting some tyrosine kinases in different neoplastic conditions, such as Bcr-Abl in chronic myeloid leukemia (21), Kit in gastrointestinal stromal tumors (2225), and PDGFR in dermatofibrosarcoma protuberans (26). Nevertheless, Krystal et al. (27) reported that STI571 failed to enhance the cytotoxicity of either carboplatin or etoposide when coadministered in small cell lung carcinoma cell lines. In the present study, we evaluated the immunohistochemical expression of Kit in a group of small cell carcinomas of the lung that underwent biopsy before and after chemotherapy. We had two main intentions: (1) to analyze whether chemotherapy effects might give rise to changes on Kit immunostaining in these tumors; and (2) to investigate whether CD117 expression in small cell lung carcinoma may be a valuable prognostic marker. Moreover, we compared the CD117 immunohistochemical expression in a series of lung tumors of different histotypes to evaluate the possible diagnostic role of this marker in the field of neoplastic lung pathology.

Our results are consistent with those reported in previous studies (6-17), confirming that a significant number of small cell lung carcinomas (21 of 27: $78 \%$ ) is positive for Kit, thus representing a possible target for a novel therapeutic approach with STI571. However, the current study shows for the first time that fewer than half of the originally CD117-positive cases maintain CD117 overexpression after standard chemotherapy. This phenomenon is probably secondary to chemotherapyinduced selection of CD117-negative neoplastic clones in relapsing small cell lung carcinomas. Kit activation in neoplastic cells is most commonly related to the acquisition of activating mutations or requires the presence of an autocrine or paracrine stimulation by its ligand $(18,34-38)$. Although no studies have explored $c$-kit locus mutation at $4 \mathrm{q} 11-$ 12 , it is interesting that genetic abnormalities involving chromosome $4 \mathrm{q}$ have been found also in small cell lung carcinoma (39). We suppose that CD117 overexpression in a significant number of small cell carcinomas of the lung could be unrelated to a true activating (gain-of-function) consti- tutional mutation, as instead shown in gastrointestinal stromal tumors (40) and more likely represents the manifestation of one of the main molecular mechanisms originally implicated in their neoplastic proliferation. Clearly, further studies mainly exploring $c$-kit sequence analysis in small cell lung carcinomas are necessary to better explain the molecular mechanisms leading to Kit activation. As also previously noted by Matsuda et al. (10) and Arber et al. (13), it is noteworthy that normal lung epithelial structures were completely unstained for CD117, thus indicating that this aberrant expression in small cell carcinomas of the lung should play an important role in promoting their pathogenesis. Taken together, all the above preclinical observations provide the scientific rationale for considering the alternative targeted therapy with STI571, strongly suggesting that at least a certain number of naive and chemoresistant small cell lung carcinomas could receive a potential benefit from this therapeutic approach. Provided that overexpression of CD117 represents the preliminary basis for using this biologic therapy, our findings may in part explain the low effectiveness recently reported by Johnson et al. (41) in their phase II study with STI571 in a small series of small cell carcinomas of the lung. Those authors, in fact, treated with STI571 19 patients with extensive stage (both chemotherapy naive and relapsed), but they tested CD117 overexpression at diagnosis in only 14 cases; moreover, in no case was the status of CD117 overexpression evaluated after tumor relapse. As well exemplified in other oncologic settings adopting biologic therapy, such as for estrogen and progesterone receptor and HER-2 status in patients with breast cancer or CD20 expression in some nonHodgkin lymphomas, immunohistochemical positivity of the neoplastic cells is one of the main criteria for a tumor to be treated with targeted therapy. Although it remains to be seen whether Kit inhibitor is really effective in this oncologic field, the frequent loss of CD117 overexpression after chemotherapy could be considered as a criterion indicating the need for a rebiopsy of the relapsed tumors before adopting this kind of treatment. This will prevent an erroneous selection of patients and forewarn of possible bias in the evaluation of clinical responses in ongoing clinical trials. By the way, in patients with refractory small cell lung carcinoma that maintain CD117 overexpression, the treatment with STI571 might be considered, especially in light of its safety and good compliance. Recently, Naeem et al. (17) reported that CD117positive small cell lung carcinomas seem to have a poorer prognosis than those that are CD117 negative. We were not able to confirm this observation, but the unbalanced number of cases in the CD117negative $(n=6)$ and -positive $(n=21)$ groups made 
it clearly difficult to drawn any statistically significant result. By the way, in our study, CD117 expression did not significantly affect patient survival and also failed to demonstrate significant differences in regard to occurrence of chemoresistant status and response to therapy.

A few works (11, 13-16) suggested that CD117 expression may be helpful in the differential diagnosis between high-grade neuroendocrine carcinomas of the lung (small cell carcinoma and large cell neuroendocrine carcinoma) on one side and other non-small cell lung tumors on the other side, the latter being generally unstained. In accordance with these studies, we confirmed that CD117 overexpression is limited to small cell carcinomas (78\%) and large cell neuroendocrine carcinomas $(60 \%)$, whereas only a few scattered tumor cells were stained in two adenocarcinomas, but not in the other analyzed histotypes. In particular, the fact that carcinoid tumors of the lung do not stain with CD117 could be instead related to their different histogenesis.

Because chemotherapy is usually used in firstline treatment of small cell carcinoma of the lung, before using CD117 as a diagnostic marker in this setting, pathologists should be aware that bioptic specimens obtained from relapses may display a complete lack of staining in a not-insignificant number of cases.

Acknowledgments: The work was funded by a grant of the Ministero dell' Istruzione, dell' Università $e$ della Ricerca (MIUR, Rome).

\section{REFERENCES}

1. Travis WD, Travis LB, Devesa SS. Lung cancer. Cancer 1995; 75:191-202.

2. Adjel AA, Marks RS, Bonner JA. Current guidelines for the management of small cell lung cancer. Mayo Clin Proc 1999; 74:809-16.

3. Johnson BE. Management of small cell lung cancer. Clin Chest Med 2002;23:225-39.

4. Spiro SG, Porter JC. Lung cancer-where are we today? Current advances in staging and nonsurgical treatment. Am J Respir Crit Care Med 2002;166:1166-96.

5. Yarden Y, Kuang WJ, Yang-Feng T, et al. Human protooncogene c-kit: a new cell surface receptor tyrosine kinase for an unidentified ligand. EMBO J 1987;6:3341-51.

6. Sekido Y, Obata Y, Ueda R, et al. Preferential expression of c-kit protooncogene transcripts in small cell lung cancer. Cancer Res 1991;51:2416-9.

7. Hibi K, Takahashi T, Sekido Y, et al. Coexpression of stem cell factor and the c-kit genes in small-cell lung cancer. Oncogene 1991;6:2291-6.

8. Natali PG, Nicotra MR, Sures I, et al. Expression of c-kit receptor in normal and transformed human nonlymphoid tissues. Cancer Res 1992;52:6139-43.

9. Rygaard K, Nakamura T, Spang-Thomsen M. Expression of the protooncogene c-met and c-kit and their ligands, hepatocyte growth factor/scatter factor and stem cell factor in SCLC cell lines and xenografts. Br J Cancer 1993;67:137-46.
10. Matsuda R, Takahashi T, Nakamura S, et al. Expression of the c-kit protein in human solid tumors and in corresponding fetal and adult normal tissues. Am J Pathol 1993;142:33946.

11. Tsuura Y, Hiraki H, Watanabe K, et al. Preferential localization of c-kit product in tissue mast cells, basal cells of skin, epithelial cells of breast, small cell lung carcinoma and seminoma/dysgerminoma in human: immunohistochemical study on formalin-fixed, paraffin-embedded tissues. Virchows Arch 1994;424:135-41.

12. Krystal GW, Hines SJ, Organ CP. Autocrine growth of small cell lung cancer mediated by coexpression of c-kit and stem cell factor. Cancer Res 1996;56:370-6.

13. Arber DA, Tamayo R, Weiss LM. Paraffin section detection of the c-kit gene product (CD117) in human tissues: value in the diagnosis of mast cell disorders. Hum Pathol 1998;28: 498-504.

14. Gibson PC, Cooper K. CD117 (KIT): a diverse protein with selective applications in surgical pathology. Adv Anat Pathol 2002;9:65-9.

15. Butnor KJ, Burchette JL, Sporn TA, et al. CD117 immunoreactivity in pleuropulmonary tumors [abstract 1317]. Mod Pathol 2002;31:316A.

16. McHale T, Litzky LA, Pasha TL, et al. Immunohistochemical detection of c-kit in neuroendocrine tumors of the lung [abstract 1352]. Mod Pathol 2002;31:324A.

17. Naeem M, Dahiya M, Clark JI, et al. Analysis of c-kit protein expression in small-cell lung carcinoma and its implication for prognosis. Hum Pathol 2002;33:1182-7.

18. Blume-Jensen $\mathrm{P}$, Hunter T. Oncogenic kinase signalling. Nature 2001;411:355-65.

19. Sekido Y, Takahashi T, Ueda R, et al. Recombinant human stem cell factor mediates chemotaxis of small-cell lung cancer cell lines aberrantly expressing the c-kit protooncogene. Cancer Res 1993;53:1709-14.

20. Plummer H, Catlett J, Leftwich J, et al. c-myc expression correlates with suppression of c-kit protooncogene expression in small cell lung cancer lines. Cancer Res 1993;53:433742.

21. Druker BJ, Talpaz M, Resta DJ, et al. Efficacy and safety of a specific inhibitor of the BCR-ABL tyrosine kinase in chronic myeloid leukemia. N Engl J Med 2001;344:1031-7.

22. Savage DG, Antman KH. Imatinib mesylate-a new oral targeted therapy. N Engl J Med 2002;346:683-93.

23. Joensuu H, Roberts PJ, Sarlomo-Rikala M, et al. Effect of the thyrosine kinase inhibitor STI571 in a patient with a metastatic gastrointestinal stromal tumor. N Engl J Med 2001;344: 1052-6.

24. Demetri GD, von Mehren M, Blanke CD, et al. Efficacy and safety of imatinib mesylate in advanced gastrointestinal stromal tumors. N Engl J Med 2002;347:472-80.

25. Heinrich MC, Blanke CD, Druker BJ, Corless CL. Inhibition of KIT tyrosine kinase activity: a novel molecular approach to the treatment of KIT-positive malignancies. J Clin Oncol 2002;20:1692-703.

26. Rubin BP, Schuetze SM, Eary JF, et al. Molecular targeting of platelet-derived growth factor B by imatinib mesylate in a patient with metastatic dermatofibrosarcoma protuberans. J Clin Oncol 2002;20:3586-91.

27. Krystal GW, Honsawek S, Litz J, Buchdunger E. The selective tyrosine kinase inhibitor STI571 inhibits small cell lung cancer growth. Clin Cancer Res 2000;6:3319-26.

28. Wang WL, Healy ME, Sattler M, et al. Growth inhibition and modulation of kinase pathways on small cell lung cancer cell lines by the novel tyrosine kinase inhibitor STI571. Oncogene 2000;19:3521-8.

29. Kijima T, Maulik G, Ma PC, et al. Regulation of cellular proliferation, cytoskeletal functions, and signal transduction 
through CXCR4 and c-Kit in small cell lung cancer cells. Cancer Res 2002;62:6304-11.

30. Zelen M. Key note address on biostatistic and data retrieval. Cancer Chemother Rep 1973;4:31-42.

31. Therasse P, Arbuck SG, Eisenhauer EA, et al. New guidelines to evaluate the response to treatment in solid tumors. J Natl Cancer Inst 2000;92:205-16.

32. Travis WD, Colby TV, Corrin B, et al. World Health Organization Pathology Panel: World Health Organization international histological typing of lung and pleural tumours. 3rd ed. Berlin: Springer-Verlag; 1999.

33. Carney DN. Lung cancer-time to move on from chemotherapy. N Engl J Med 2002;346:126-8.

34. Sekido Y, Fong KM, Minna JD. Progress in understanding the molecular pathogenesis of human lung cancer. Biochim Biophys Acta 1998;1378:F21-59.

35. Wistuba II, Gazdar AF, Minna JD. Molecular genetics of small cell lung carcinoma. Semin Oncol 2001;28(Suppl 4):3-13.
36. Arteaga CL, Khuri F, Krystal G, Sebti S. Overview of rationale and clinical trials with signal transduction inhibitors in lung cancer. Semin Oncol 2002;29(Suppl 4):15-26.

37. Dy GK, Adjei AA. Novel targets for lung cancer therapy: part I. J Clin Oncol 2002;20:2881-94.

38. Fong KM, Minna JD. Molecular biology of lung cancer: clinical implications. Clin Chest Med 2002;23:83-101.

39. Shivapurkar N, Virmani AK, Witsuba II, et al. Deletions of chromosome 4 at multiple sites are frequent in malignant mesothelioma and small cell lung carcinoma. Clin Cancer Res 1999;59:1973-9.

40. Heinrich MC, Rubin BP, Longley JB, Fletcher JA. Biology and genetic aspects of gastrointestinal stromal tumors: KIT activation and cytogenetic alterations. Hum Pathol 2002;33:48495.

41. Johnson BE, Fisher B, Fisher T, et al. Phase II study of STI571 (Gleevec ${ }^{\circledR}$ ) for patients with small cell lung cancer [abstract 1171]. Proc Am Soc Clin Oncol 2002;21:293a. 